International Journal of Theoretical Physics, Vol. 43, No. 6, 2004, 1363-1369. DOI: 10.1023/B:IJTP.0000048621.17418.bb

\title{
Central Elements of Effect Algebras
}

\author{
Josef Tkadlec ${ }^{1}$ \\ Received November 15, 2001 \\ Central elements of an effect algebra can be characterized by means of a weak \\ form of distributivity and a maximality property. We give examples when these \\ two conditions are fulfilled.
}

Central elements and the center (the set of all central elements) play an important role in quantum structures - they represent the "classical part" of a given model. Considering the axiomatics of quantum structures, it is important to know the impact of various conditions on the size of the center. In particular, there is a lot of results of the type that a quantum structure with some properties has to be a Boolean algebra - see, e.g., Navara and Pták (1989), Müller et al. (1992), Pulmannová and Majerník (1992), Müller (1993), Pulmannová (1993), Pták and Pulmannová (1994), Tkadlec (1994), Dvurečenskij and Länger (1995), Tkadlec (1995), Navara (1997). All the results mentioned above were generalized by Tkadlec (1997) by introducing two new classes of orthomodular posets. Here we present a generalization of the latter results in two direction: 1) we consider more general structures than orthomodular posets, 2) we consider a given element instead of the whole structure.

\section{BASIC NOTIONS AND PROPERTIES}

Let us summarize some basic notions and properties of effect algebras. For proofs and details see, e.g., Foulis and Bennett (1994), Greechie et al. (1995).

Definition 1.1. An effect algebra is an algebraic structure $(E, 0,1, \oplus)$ such that $E$ is a set, 0 and 1 are different elements of $E$ and $\oplus$ is a partial binary operation on $E$ such that for every $a, b, c \in E$ the following conditions hold (the equalities mean also "if one side exists then the other side exists"):

\footnotetext{
${ }^{1}$ Department of Mathematics, Faculty of Electrical Engineering, Czech Technical University, 16627 Prague, Czech Republic; e-mail: tkadlec@fel.cvut.cz.
} 
(1) $a \oplus b=b \oplus a$ (commutativity),

(2) $(a \oplus b) \oplus c=a \oplus(b \oplus c)$ (associativity),

(3) for every $a \in E$ there is a unique $a^{\prime} \in E$ such that $a \oplus a^{\prime}=1$ (orthosupplement),

(4) $a=0$ whenever $a \oplus 1$ is defined (zero-unit law).

For simplicity, we use the notion $E$ for an effect algebra. A partial ordering on an effect algebra $E$ is defined by $a \leq b$ iff there is a $c \in E$ such that $b=a \oplus c$; such an element $c$ is unique (if it exists) and is denoted by $b \ominus a .0(1$, resp.) is the least (the greatest, resp.) element of $E$ with respect to this partial ordering. An orthogonality relation on $E$ is defined by $a \perp b$ iff $a \oplus b$ exists (i.e., iff $a \leq b^{\prime}$ ). It can be shown that $a \oplus 0=a$ for every $a \in E$ and that a cancellation law is valid: for every $a, b, c \in E$ with $a \oplus b \leq a \oplus c$ we have $b \leq c$.

Definition 1.2. An orthoalgebra is an effect algebra $E$ such that for every $a \in$ E:

(4') $a=0$ whenever $a \oplus a$ is defined (consistency).

Assuming conditions (1)-(3) from the definition of an effect algebra, the condition (4') is stronger than condition (4), hence we can define an orthoalbegra by conditions (1)-(3) and (4'). Other characterizations of orthoalgebras among effect algebras: $a \wedge a^{\prime}=0$ for every $a$; the orthosupplementation is orthocomplementation; $a \oplus b$ is a minimal upper bound for orthogonal $a, b$.

Definition 1.3. An orthomodular poset is an effect algebra $E$ such that for every $a, b, c \in E:$

(5) $(a \oplus b) \oplus c$ is defined whenever $a \oplus b, b \oplus c$ and $c \oplus a$ are defined (coherence).

Obviously, if $a \perp b$ and $a \vee b$ exists in an effect algebra, then $a \vee b \leq a \oplus b$. The reverse inequality need not be true.

Definition 1.4. An element $a$ of an effect algebra $E$ is principal, if $b \oplus c \leq a$ for every $b, c \in E$ such that $b, c \leq a$ and $b \perp c$.

If $a$ is a principal element, then $a \wedge a^{\prime}=0$ and the interval $[0, a]$ is an effect algebra with the greatest element $a$ and the partial operation given by restriction of $\oplus$ to $[0, a]$ — the orthosupplement operation is given by $b \mapsto\left(b \oplus a^{\prime}\right)^{\prime}$. Moreover:

Theorem 1.5. An effect algebra is an orthomodular poset iff every its element is principal.

Our interest will be concentrated on central elements.

Definition 1.6. An element $a$ of an effect algebra $E$ is central, if 
(1) $a$ and $a^{\prime}$ are principal,

(2) for every $b \in E$ there are $b_{1}, b_{2} \in E$ such that $b_{1} \leq a, b_{2} \leq a^{\prime}$ and $b=b_{1} \oplus b_{2}$.

The center $C(E)$ of $E$ is the set of all central elements of $E$.

The center of an effect algebra $E$ is a sub-effect algebra of $E$ and forms a Boolean algebra. The decomposition property of central elements (condition (2) of Definition 1.6) can be formulated by the following way: $b=(b \wedge a) \oplus\left(b \wedge a^{\prime}\right)$.

\section{RESULTS}

Let us present the main result, which generalizes Theorem 4.2 from Tkadlec (1997).

Theorem 2.1. Let $E$ be an effect algebra. Then $a \in E$ is central iff the following conditions hold:

(1) a and $a^{\prime}$ are principal,

(2) $b=0$ whenever $b \in E$ with $b \wedge a=b \wedge a^{\prime}=0$,

(3) $[0, a] \cap[0, b],\left[0, a^{\prime}\right] \cap[0, b]$ have maximal elements for every $b \in E$.

Proof: The necessity of all conditions is obvious - the first is condition (1) of Definition 1.6, the second is a weaker form of condition (2) of Definition 1.6, the third follows from the fact that for a central element $a$ and for an arbitrary $b \in E$ the infima $b \wedge a, b \wedge a^{\prime}$ exist.

Let us prove the sufficiency, i.e., condition (2) of Definition 1.6. Let $b \in E$. Let us denote by $b_{1}$ a maximal element of $[0, a] \cap[0, b]$ and by $b_{2}$ a maximal element of $\left[0, a^{\prime}\right] \cap\left[0, b \ominus b_{1}\right]$ and consider the element $c=\left(b \ominus b_{1}\right) \ominus b_{2}$. Let us take an arbitrary $d \leq c \wedge a$. Since $d \leq c \leq b \ominus b_{1}$, we obtain that $d \oplus b_{1}$ exists and $d \oplus b_{1} \leq b$. Since $d, b_{1} \leq a$ and $a$ is principal, $d \oplus b_{1} \leq a$. Since $b_{1}$ is a maximal element of $[0, a] \cap[0, b], d \oplus b_{1}=b_{1}$ and therefore $d=0$, i.e., $c \wedge a=0$. Analogously, $c \wedge a^{\prime}=0$ and, according to assumption (2), $c=0$. Therefore $b=b_{1} \oplus b_{2}$ and the condition (2) from Definition 1.6 is proved.

The following examples show that no condition from Theorem 2.1 can be omitted.

Examples 2.2. 1) Let $E$ be the real interval $[0,1]$ with $a \oplus b=a+b$ if $a+b \leq 1$. Then the partial ordering is the standard (total) ordering of real numbers and $a^{\prime}=1-a$ for every $a \in E$. The condition (1) of Theorem 2.1 is fulfilled only for $a \in\{0,1\}$ while the others are fulfilled for every $a \in E$.

2) Let $E$ be the family od even-element subsets of a 4-element set $X, 0=\emptyset$, $1=X$ and $a \oplus b=a \cup b$ if $a$ and $b$ are disjoint. The condition (2) of Theorem 2.1 is fulfilled only for $a \in\{0,1\}$ while the others are fulfilled for every $a \in E$. 
3) Let $X_{1}, X_{2}, X_{3}, X_{4}$ be pairwise disjoint infinite sets, $X$ be their union, $E$ consists of $X_{1} \cup X_{2}, X_{2} \cup X_{3}, X_{3} \cup X_{4}, X_{4} \cup X_{1}, \emptyset, X$ and of all subsets of $X$ with finite symmetric difference with some of these sets. Let $a \oplus b=a \cup b$ if $a$ and $b$ are disjoint. The condition (3) of Theorem 2.1 is fulfilled only for $a$ finite or cofinite subsets of $X$ while the others are fulfilled for every $a \in E$.

Due to Theorem 1.5, we can omit condition (1) of Theorem 2.1 for orthomodular posets. If we apply the above theorem for every element of a given effect algebra, we obtain the following corollary:

Corollary 2.3. Let $E$ be an effect algebra such that for every $a, b \in E$ the following conditions hold:

(1) a is principal,

(2) $b=0$ whenever $b \wedge a=b \wedge a^{\prime}=0$,

(3) $[0, a] \cap[0, b]$ has a maximal element.

Then $E$ is a Boolean algebra.

Again, for orthomodular posets we may omit the first condition and obtain thus exactly the result of Tkadlec (1997).

Properties used in Theorem 2.1 might be derived from other properties. First, let us introduce some notions.

Definition 2.4. Let $E$ be an effect algebra. A state $s$ on $E$ is a mapping $s: E \rightarrow$ $[0,1]$ such that:

(1) $s(1)=1$,

(2) $s(a \oplus b)=s(a)+s(b)$ whenever $a \oplus b$ is defined.

A state $s$ is Jauch-Piron if for every $a, b \in E$ with $s(a)=s(b)=1$ there is a $c \in E$ such that $c \leq a, b$ and $s(c)=1$.

A set $S$ of states on $E$ is unital, if for every $a \in E \backslash\{0\}$ there is a state $s \in S$ such that $s(a)=1$.

Obviously, for every state $s$ we have $s(0)=0, s\left(a^{\prime}\right)=1-s(a)$ for every $a \in E, s(a) \leq s(b)$ for $a \leq b$. By a two-valued state we mean a state with values in $\{0,1\}$.

The condition (2) of Theorem 2.1 is a week form of distributivity - it can be reformulated by the following way: $b \wedge\left(a \vee a^{\prime}\right)=(b \wedge a) \vee\left(b \wedge a^{\prime}\right)$ whenever $b \wedge a=b \wedge a^{\prime}=0$. It is a consequence of a large number of various properties usually stating that we have "sufficiently enough" of "sufficiently good" states.

Proposition 2.5. Let $E$ be an effect algebra, $a \in E$ and at least one of the following conditions hold:

(1) $a$ is principal and $b \perp a, a^{\prime}$ for every $b \in E$ with $b \wedge a=b \wedge a^{\prime}=0$. 
(2) There is $a k \in\left[\frac{1}{2}, 1\right]$ and a set $S$ of states on $E$ such that for every $b \in E \backslash\{0\}$ with $b \wedge a=b \wedge a^{\prime}=0$ the following holds:

(a) there is a state $s \in S$ such that $s(b) \geq k(s(b)>k$, resp.),

(b) $s(a)+s(b)<\frac{1}{2}+k, s\left(a^{\prime}\right)+s(b)<\frac{1}{2}+k\left(s(a)+s(b) \leq \frac{1}{2}+k\right.$, $s\left(a^{\prime}\right)+s(b) \leq \frac{1}{2}+k$, resp.) whenever $s \in S$ with $s(b) \geq k(s(b)>k$, resp.).

Then the condition (2) of Theorem 2.1 is fulfilled.

Proof: (1) Let $b \in E$ such that $b \wedge a=b \wedge a^{\prime}=0$. Then $a \oplus b$ exists and $b \perp a^{\prime}$, i.e., $b \leq a$. Since $a$ is principal, $a \oplus b \leq a=a \oplus 0$ and therefore $b=0$.

(2) Let $b \in E \backslash\{0\}$ such that $b \wedge a=b \wedge a^{\prime}=0$ and let us seek a contradiction. There is an $s \in S$ such that $s(b) \geq k\left(s(b)>k\right.$, resp.) and $s(a)+s(b)<\frac{1}{2}+k$, $s\left(a^{\prime}\right)+s(b)<\frac{1}{2}+k$ ( $\leq$ in both inequalities, resp.). Adding these two inequalities and using the equality $s(a)+s\left(a^{\prime}\right)=1$ we obtain $s(b)<k(s(b) \leq k$, resp. $)$-a contradiction.

Let us remark that the assumption that $a$ is principal in condition (1) of the last proposition is required in condition (1) of Theorem 2.1. A special case of (2) we obtain for $S$ be a "unital set of two-valued Jauch-Piron states". For other concepts (e.g. for various kinds of subadditive states) see Tkadlec (1997).

The condition (3) of Theorem 2.1 is also a consequence of various properties.

Proposition 2.6. Let $E$ be an effect algebra, $a \in E$ and at least one of the following conditions hold:

(1) $b \wedge a, b \wedge a^{\prime}$ exist for every $b \in E$ (special case: $E$ is a lattice),

(2) $[0, a],\left[0, a^{\prime}\right]$ are closed under suprema of orthogonal elements (special case: $E$ is orthocomplete in the lattice sence) and $E$ is an orthoalgebra.

(3) There is a countable unital set of states on $E$ and every state on $E$ is Jauch-Piron.

Then the condition (3) of Theorem 2.1 is fulfilled.

Proof: Let $b \in E$, we will prove the existence of a maximal element of $[0, a] \cap$ $[0, b]$ (for $a^{\prime}$ we can proceed analogously).

(1) $a \wedge b$ is the greatest (hence a maximal) element of $[0, a] \cap[0, b]$.

(2) There is a maximal set $O$ of mutually orthogonal elements from $[0, a] \cap$ $[0, b]$. Then $c=\bigvee O \in E$ is a maximal element of $[0, a] \cap[0, b]$. Indeed, if $d \in[0, a] \cap[0, b]$ with $d \geq c$, then $e=d \ominus c \in[0, a] \cap[0, b]$ and $e \perp c$. Due to the maximality of $O, e \in O$ and therefore $e \perp e$. Since $E$ is an orthoalgebra, we obtain $e=0$ and $d=c$, i.e., $c$ is a maximal element of $[0, a] \cap[0, b]$.

(3) If $[0, a] \cap[0, b]=\{0\}$ then the maximal element of $[0, a] \cap[0, b]$ is 0 . Let us suppose that $[0, a] \cap[0, b] \neq\{0\}$ and let us denote by $S$ a countable unital set of states on $E$. Then the set $S_{a, b}=\{s \in S ; s(a)=s(b)=1\}$ is nonempty and countable. Let $s_{0}$ be a $\sigma$-convex combination (with nonzero coefficients) of 
all states in $S_{a, b}$. Then $s_{0}(a)=s_{0}(b)=1$. Since the state $s_{0}$ is Jauch-Piron, there is a $c \in[0, a] \cap[0, b]$ such that $s_{0}(c)=1$. It remains to prove that $c$ is a maximal element of $[0, a] \cap[0, b]$. Indeed, if $d \in[0, a] \cap[0, b]$ with $d \geq c$ then $e=d \ominus c \in[0, a] \cap[0, b]$ and $e \perp c$. Hence $s_{0}(e)=0$ and therefore there is no state $s \in S$ such that $s(e)=1$. Due to the unitality of $S, e=0$ and therefore $d=c$.

The condition (3) of the above Proposition is weaker than the condition "the set of states on $E$ is a countable unital set of Jauch-Piron states":

Example 2.7. Let $E$ be the Boolean algebra of open subsets of the real interval $[0,1]$. Every state on $E$ is Jauch-Piron, the set of states is uncountable (for every $x \in[0,1]$ there is a two-valued stated carried by $x: s_{x}(a)=1$ iff $x \in a$ ) but there is a countable unital set of states (carried by rational numbers).

\section{ACKNOWLEDGMENTS}

The author gratefully acknowledges the support of Grant No. 201/00/0331 of the Grant Agency of the Czech Republic and of Project No. MSM 210000010 of the Ministry of Education of the Czech Republic.

\section{REFERENCES}

Dvurečenskij, A., Länger, H. (1995). Bell-type inequalities in orthomodular lattices I, Inequalities of order 2, Internat. J. Theoret. Phys. 34, 995-1024.

Foulis, D. J., Bennett, M. K. (1994). Effect algebras and unsharp quantum logics, Foundations of Physics, 24, 1331-1352.

Greechie, R. J., Foulis, D., Pulmannová, S. (1995). The center of an effect algebra, Order, 12, 91-106.

Müller, V. (1993). Jauch-Piron states on concrete quantum logics, Internat. J. Theoret. Phys. 32, 433-442.

Müller, V., Pták, P., Tkadlec, J. (1992) Concrete quantum logics with covering properties, Internat. J. Theoret. Phys. 31 (1992), 843-854.

Navara, M. (1997). On generating finite orthomodular sublattices, Tatra Mt. Math. Publ. 10, 109-117.

Navara, M., Pták, P. (1989). Almost Boolean orthomodular posets, J. Pure Appl. Algebra 60 (1989), 105-111.

Pták, P., Pulmannová, S. (1994). A measure-theoretic characterization of Boolean algebras among orthomodular lattices, Comment. Math. Univ. Carolin. 35, 205-208.

Pulmannová, S. (1993). A remark on states on orthomodular lattices, Tatra Mt. Math. Publ. 2, 209-219.

Pulmannová, S., Majerník, V. (1992). Bell inequalities on quantum logics, J. Math. Phys. 33, 2173-2178.

Tkadlec, J. (1994). Boolean orthoposets - concreteness and orthocompleteness, Math. Bohem. 119, $123-128$.

Tkadlec, J. (1995). Subadditivity of states on quantum logics, Internat. J. Theoret. Phys. 34 (1995), 1767-1774.

Tkadlec, J. (1997). Conditions that force an orthomodular poset to be a Boolean algebra, Tatra Mt. Math. Publ., 10, 55-62. 